\title{
JEAN DESCLOUX
}

NABIL NASSIF

JACQUES RAPPAZ

\section{On spectral approximation. Part 2. Error estimates for the Galerkin method}

RAIRO - Analyse numérique, tome 12, no 2 (1978), p. 113-119.

$<$ http://www.numdam.org/item?id=M2AN_1978_12_2_113_0>

(C) AFCET, 1978, tous droits réservés.

L'accès aux archives de la revue « RAIRO - Analyse numérique » implique l'accord avec les conditions générales d'utilisation (http://www.numdam.org/ legal.php). Toute utilisation commerciale ou impression systématique est constitutive d'une infraction pénale. Toute copie ou impression de ce fichier doit contenir la présente mention de copyright.

\section{Numdam}




\title{
ON SPECTRAL APPROXIMATION \\ PART 2. ERROR ESTIMATES \\ FOR THE GALERKIN METHOD (*)
}

\author{
by Jean Descloux $\left({ }^{\mathbf{1}}\right)$, Nabil Nassif $\left({ }^{2}\right)$ and Jacques RapPaz $\left({ }^{\mathbf{1}}\right)$ \\ Communiqué par P.-A. RaviarT
}

\begin{abstract}
One considers an isolated eigenvalue A of finite multiplicity of an operator $\boldsymbol{A}$ which is approximated by a Galerkin method. Using Osborn's technics, one derives several error estimates for $\lambda$.
\end{abstract}

\section{SITUATION AND RESULTS}

In part 1 of this paper [3], we have been concerned with the problem of convergence in spectral approximation; since the theory we have developped has received concrete applications for non compact operators only in connection with the Galerkin method, we shall now restrict ourself to this case.

Let $X$ be a complex Banach space of norm \|\| and $\left\{X_{h}\right\}$ be a sequence of finite dimensional subspaces of $X$. One gives two continuous sesquilinear forms $a$ and $b$ on $X$ and one supposes a coercive. Then, by Lax-Milgram, one can define the continuous operators $A: X \rightarrow X$ and $A_{h}: X_{h} \rightarrow X_{h}$ by

$$
a(A u, v)=b(u, v), \quad \forall u, v \in X, \quad a\left(A_{h} u, v\right)=b(u, v), \quad \forall u, v \in X_{h} .
$$

All along this paper we shall suppose that the two following conditions are satisfied (see [3]):

$$
\text { P1: } \lim _{h \rightarrow 0}\left\|\left.\left(A-A_{h}\right)\right|_{X_{h}}\right\|=0 ; \quad \text { P2: } \forall x \in X, \lim _{h \rightarrow 0} \inf _{x_{h} \in X_{h}}\left\|x-x_{h}\right\|=0 .
$$

Let $\lambda \in \mathbf{C}$ be an isolated eigenvalue of $A$ of finite algebraic multiplicity $m$; since $\mathrm{a}$ is coercive $\lambda \neq 0$ and there exists a closed disc $\Delta$ of center $\lambda$ and boundary $\Gamma$ such that $0 \notin \Delta$ and $\Delta \cap \sigma(A)=\{\lambda\}$ where $\sigma(A)$ denotes the spectrum of $A$. Let $\mu_{1 h}, \ldots, \mu_{m(h), h}$ be the eigenvalues of $A_{h}$, repeated following their algebraic multiplicities and contained in $\Delta$. In [3], section 2 ,

(*) Manuscrit reçu le 10 juin 1977.

(1) Département de Mathématiques, École Polytechnique fédérale de Lausanne, Suisse.

(2) Department of Mathematics, American University of Beirut, Liban. 
we have proved:

a) $m(h)=m$ for $h$ small enough;

b) $\lim _{h \rightarrow 0} \mu_{i h}=\lambda, i=1,2, \ldots, m$.

The purpose of this part 2 of our paper is to give estimates of $\lambda$ by the $\mu_{i h}$ 's. In fact, we shall adapt to the situation described above Osborn's method [5]; note that, independently of the fact that $A_{h}$ is a Galerkin approximation, we have simplified the presentation of Osborn's main argument and strengthened his results. See also Grigorieff [4].

At this point, we recall some standard notations. For an operator $D$, $R_{z}(D)=(z-D)^{-1}$ is the resolvent operator. Let $Y$ and $Z$ be closed subspaces of $X$; then for $x \in X$,

$$
\delta(x, Z)=\inf _{z \in Z}\|x-z\|, \quad \delta(Y, Z)=\sup _{\substack{y \in Y \\\|y\|=1}} \delta(y, Z)
$$

and

$$
\hat{\delta}(Y, Z)=\max (\delta(Y, Z), \delta(Z, Y)) \text {. }
$$

Let us also open a short parenthesis on duality. Let $X^{*}$ be the adjoint space of $X$, i. e. the set of antilinear continuous forms on $X$. By Lax-Milgram, the operator $C: X^{*} \rightarrow X$ defined by the relation $a(v, C \varphi)=\bar{\varphi}(v), \forall v \in X$, $\varphi \in X^{*}$, is an isomorphism between $X^{*}$ and $X$ which allows to identify these two spaces. With this identification if $D: X \rightarrow X$ is a bounded linear operator, its adjoint $D^{*}: X \rightarrow X$ will be characterized by the relation $a(D u, v)=a\left(u, D^{*} v\right), \forall u, v \in X$; one verifies also immediately the relation $\left\|D^{*}\right\| \leqq\|C\| \cdot\left\|C^{-1}\right\| \cdot\|D\|$.

We need, for the following, to introduce some further operators. $\Pi_{h}: X \rightarrow X$ is the projector with range $X_{h}$ defined by the relation $a\left(\Pi_{h} u-u, v\right)=0$, $\forall v \in X_{h}$. One has $A_{h}=\left.\Pi_{h} A\right|_{X_{h}}$ and we set $B_{h}=\Pi_{h} A \Pi_{h}: X \rightarrow X$; exept for zero, $B_{h}$ has the same spectrum as $A_{h}$ and the same corresponding invariant subspaces. $E=(2 \Pi i)^{-1} \int_{\Gamma} R_{z}(A) d z$ is the spectral projector of $A$ relative to $\lambda$ and, for $h$ small enough, $F_{h}=(2 \Pi i)^{-1} \int_{\Gamma} R_{z}\left(B_{h}\right) d z$ is the spectral projector of $B_{h}$ relative to $\mu_{1 h}, \ldots, \mu_{m h}$. Now consider the adjoints of these operators as defined above. $A^{*}$ has the isolated eigenvalue $\bar{\lambda}$ of algebraic multiplicity $m ; \Pi_{h}^{*}$ will be the projector with range $X_{h}$ satisfying the relation $a\left(v, \Pi_{h}^{*} u-u\right)=0, \forall v \in X_{h} ; E^{*}$ and $F_{h}^{*}$ will be the spectral projectors of $A^{*}$ and $B_{h}^{*}=\Pi_{h}^{*} A^{*} \Pi_{h}^{*}$ associated respectively to $\bar{\lambda}$ and to the set $\bar{\mu}_{1 h}, \ldots, \bar{\mu}_{m h}$; 
they will satisfy the relations

$$
E^{*}=(2 \Pi i)^{-1} \int_{\bar{\Gamma}} R_{z}\left(A^{*}\right) d z \quad \text { and } \quad F_{h}^{*}=(2 \Pi i)^{-1} \int_{\bar{\Gamma}} R_{z}\left(B_{h}^{*}\right) \dot{d} z
$$

where $\bar{\Gamma}$ is the conjugate circle of $\Gamma$ (positively oriented).

In applications $E(X)$ and $E^{*}(X)$, the $m$-dimensional invariant subspaces of $A$ and $A^{*}$ corresponding to $\lambda$ and $\bar{\lambda}$, will be often composed of smooth functions so that it is reasonable to introduce the quantities

$$
\gamma_{h}=\delta\left(E(X), X_{h}\right), \quad \gamma_{h}^{*}=\delta\left(E^{*}(X), X_{h}\right) .
$$

We can now state the results.

THEOREM $1:$ There exists a constant $c$, independent of $h$ such that

$$
\hat{\delta}\left(F_{h}(X), E(X)\right) \leqq c \gamma_{h} ; \quad \hat{\delta}\left(F_{h}^{*}(X), E^{*}(X)\right) \leqq c \gamma_{h}^{*} .
$$

In section 2, we shall show that $\left.\mathrm{F}_{h}\right|_{E(X)}$ defines for $h$ small enough, a bijection between $E(X)$ and $F_{h}(X)$; let $\Lambda_{h}$ be this bijection; $\hat{A}=\left.A\right|_{E(X)}$ and $\hat{B}_{h}=\Lambda_{h}^{-1} B_{h} \Lambda_{h}$ will be considered as operators in $E(X) ; \hat{A}$ has the eigenvalue $\lambda$ of algebraic multiplicity $m$ and $\hat{B}_{h}$ has the eigenvalues $\mu_{1 h}, \ldots, \mu_{m h}$.

THEOREM 2: There exists a constant $c$, independent of $h$ such that

$$
\left\|\hat{A}-\hat{B}_{h}\right\|_{E(X)} \leqq c \gamma_{h} \gamma_{h}^{*} .
$$

By the choice of a basis in $E(X)$, theorem 2 reduces our original task to a pure matricial problem. Let $f$ be a holomorphic function defined in a neighborhood of $\lambda$; writting $f(\hat{A})$ and $f\left(\hat{B}_{h}\right)$ by the mean of Dunford integrals, one verifies immediately that

$$
\| f\left(\hat{A)}-f\left(\hat{B}_{h}\right)\left\|_{E(X)} \leqq c\right\| \hat{A}-\hat{B}_{h} \|_{E(X)}\right.
$$

where $c$ depends on $f$ but not on $h$; using the classical properties of traces and determinants, one obtains theorem $3 a, b$; theorem $3 c, d$ is a direct application of results quoted in [7], pp. $80-81$; here $\alpha$ is the ascent of the eigenvalue $\lambda$ of $\hat{A}, \beta$ is the number of Jordan blocs of the canonical form of $\hat{A}$. 
THEOREM 3: There exists a constant $c$, independent of $h$ such that for $h$ small enough:

a)

$$
\left|f(\lambda)-\frac{1}{m} \sum_{i=1}^{m} f\left(\mu_{i h}\right)\right| \leqq c \gamma_{h} \gamma_{h}^{*},
$$

b)

$$
\left|f^{m}(\lambda)-\prod_{i=1}^{m} f\left(\mu_{i h}\right)\right| \leqq c \gamma_{h} \gamma_{h}^{*}
$$

c)

d)

$$
\max _{i=1 \ldots m}\left|\lambda-\mu_{i h}\right| \leqq c\left(\gamma_{h} \gamma_{h}^{*}\right)^{1 / \alpha},
$$

$$
\min _{i=1 \ldots m}\left|\lambda-\mu_{i h}\right| \leqq c\left(\gamma_{h} \gamma_{h}^{*}\right)^{\beta / m}
$$

REMARKS : 1) In his original work, in a difference context, Osborn [5] has obtained theorem $3 a$ for $f(z)=z$ and theorem $3 c$; in another context also Chatelin [1] proves, theorem $3 a$ for $f(z)=z$.

2) For $f(z)=1 / z$, theorem $3 a$ gives an estimate of $1 / \lambda$ by the arithmetic mean of the $1 / \mu_{i n}$ 's; the result has been already obtained by [2]; we are indebted to Chatelin who showed us that it can also be deduced by Osborn's method.

In order to illustrate this theorem, we consider the example developped in section 4 of part 1 of this paper [3]; one can prove by Rappaz' method of elimination used in [6] the existence of an infinite number of isolated eigenvalues of finite multiplicities; by supposing the coefficients $\alpha, \beta, \ldots$ sufficiently smooth, one verifies that the corresponding eigensubspaces are subsets of $H^{2} \times\left(H^{1}\right)^{2}$; consequently $\gamma_{h}=O(h), \gamma_{h}^{*}=O(h)$ and the estimates of theorem $3 a, b$ are of order $h^{2}$.

We conclude this section by stating a very elementary result for the selfadjoint case. We suppose that the forms $a$ and $b$ are hermitian. Because of its coercivity, a is a scalar product for which $X$ is a Hilbert space with norm $\|x\|_{a}^{2}=a(x, x)$; then $A, B_{h}$ and $\Pi_{h}$ become hermitian. Let $v$ be an eigenvalue of $A$, which is not supposed isolated or of finite multiplicity, and $G$ be the corresponding eigensubspace. For the distance $\delta\left(v, \sigma\left(B_{h}\right)\right)$ from $v$ to the spectrum $\sigma\left(B_{h}\right)$ of $B_{h}$, one gets the estimate

$$
\begin{aligned}
\delta\left(v, \sigma\left(B_{h}\right)\right) & =\inf _{\substack{y \in X \\
\|y\|=1}}\left\|\left(B_{h}-v\right) y\right\|_{a} \leqq \inf _{\substack{y \in X_{h} \\
\|y\|=1}}\left\|\left(B_{h}-v\right) y\right\|_{a} \\
& \leqq \inf _{\substack{y \in X_{h} \\
\|y\|=1}}\|(A-v) y\|_{a}=\inf _{\substack{y \in X_{h} \\
\|y\|=1}}\|(A-v)(y-x)\|_{a}, \quad \forall x \in G,
\end{aligned}
$$

R.A.I.R.O. Analyse numérique/Numerical Analysis 
i. e., since the norms $\|\cdot\|$ and $\|\cdot\|_{a}$ are equivalent:

$$
\delta\left(v, \sigma\left(B_{h}\right)\right) \leqq c \inf _{\substack{x \in G \\\|x\|=1}} \delta\left(x, X_{h}\right), \quad c \text { independent of } h \text { and } v .
$$

Remarks: 1) We have obtained the estimate (1) without supposing P1 or P2.

2) Examples show that it is not possible to replace the right member of (1), by $c\left\{\inf \delta\left(x, X_{h}\right)\right\}^{1+\varepsilon}, \varepsilon>0$.

$$
\underset{\| x \in G}{\|x\|=1}
$$

\section{PROOFS}

In this section we prove theorem 1 and 2 . We use the definitions and notations of section 1 and we suppose hypotheses P1 and P2. $c$ will denote a "generic" constant.

We first recall a well-known result. Since a is continuous and coercive, the projectors $\Pi_{h}$ are bounded uniformly with respect to $h$ and there exists a constant $c$ such that $\left\|x-\Pi_{h} x\right\| \leqq c \delta\left(x, X_{h}\right), \forall x \in X$; the $\Pi_{h}^{*}$ 's possess the same properties.

Lemma 1 of section 2 of [3] shows that P1 implies the inequality sup $\left\|R_{z}\left(A_{h}\right) x\right\| \leqq c, \forall z \in \Gamma$ for $h$ small enough, $c$ independent of $h$. $x \in X_{h}$
$\|x\|=1$

We extend this result to $B_{h}$ and $B_{h}^{*}$.

LEMMA 1: There exists $h_{0}>0$ and c such that

and

$$
\left\|R_{z}\left(B_{h}\right)\right\| \leqq c, \quad h<h_{0}, \quad z \in \Gamma
$$

$$
\left\|R_{z}\left(B_{h}^{*}\right)\right\| \leqq c, \quad h<h_{0}, \quad z \in \bar{\Gamma} .
$$

Proof: Since $R_{\bar{z}}\left(B_{h}^{*}\right)=\left(R_{z}\left(B_{h}\right)\right)^{*}$ we need to prove only the first statement; since $B_{h}$ is compact it suffices to verify that $\left\|\left(z-B_{h}\right) x\right\| \geqq c\|x\|, \forall x \in X$, $z \in \Gamma$. Taking in account the fact that $0 \notin \Gamma$ one has

$$
\begin{aligned}
& \|x\| \leqq\left\|\Pi_{h} x\right\|+\left\|x-\Pi_{h} x\right\| \\
& \quad \leqq c\left\|\left(z-B_{h}\right) \Pi_{h} x\right\|+|z|^{-1} \mid i\left(z-B_{h}\right)\left(x-\Pi_{h} x\right)\|\leqq c\|\left(z-B_{h}\right) x \| .
\end{aligned}
$$

We note that we shall not use any more P1 explicitely. Consequently, in the proofs of lemma 3 and theorem 1, the statements for the adjoints operators are obtained in the same way as for the direct operators.

We omit the proof of the following trivial:

LEMMA $2:$ Let $Y$ and $Z$ be two subspaces of $X$ with the same finite dimension; 1 et $P: Y \rightarrow Z$ be a linear operator such that $\|P y-y\| \leqq 0.5\|y\|, \forall y \in Y$. 
Then $P$ is bijective,

$$
\left\|P^{-1} z\right\| \leqq 2\|z\|, \quad \forall z \in Z
$$

and

$$
\sup _{\substack{z \in Z \\\|z\|=1}}\left\|P^{-1} z-z\right\| \leqq 2 \sup _{\substack{y \in Y \\\|y\|=1}}\|P y-y\| .
$$

LEMMA 3:

$$
\begin{gathered}
\left\|\left.\left(E-F_{h}\right)\right|_{E(X)}\right\| \leqq c\left\|\left.\left(A-B_{h}\right)\right|_{E(X)}\right\| \leqq c \gamma_{h}, \\
\left\|\left.\left(E^{*}-F_{h}^{*}\right)\right|_{E^{*}(X)}\right\| \leqq c\left\|\left.\left(A^{*}-B_{h}^{*}\right)\right|_{E^{*}(X)}\right\| \leqq c \gamma_{h}^{*} .
\end{gathered}
$$

Proof: For $h$ small enough, by lemma 1, one has

$$
\begin{aligned}
\left\|\left.\left(E-F_{h}\right)\right|_{E(X)}\right\| & \leqq\left.(2 \Pi)^{-1} \int_{\Gamma}\left\|R_{z}\left(B_{h}\right)\right\| \cdot||\left(A-B_{h}\right) R_{z}(A)\right|_{E(X)} \| \cdot|d z| \\
\leqq c\left\|\left.\left(A-B_{h}\right)\right|_{E(X)}\right\| & \\
\left\|\left.\left(A-B_{h}\right)\right|_{E(X)}\right\| & \leqq\left\|\left.\left(I-\Pi_{h}\right) A\right|_{E(X)}\right\|+\left\|\left.\Pi_{h} A\left(I-\Pi_{h}\right)\right|_{E(X)}\right\| \\
& \leqq c\left\|\left.\left(I-\Pi_{h}\right)\right|_{E(X)}\right\| \leqq c \gamma_{h} .
\end{aligned}
$$

Proof of theorem 1: Lemma 3 implies that $\delta\left(E(X), F_{h}(X)\right) \leqq c \gamma_{h}$. Set, as in Section 1, $\Lambda_{h}=\left.F_{h}\right|_{E(X)}: E(X) \rightarrow F_{h}(X)$; for $h$ small enough $E(X)$ and $F_{h}(X)$ have the same dimension $m$; on the other side P2 implies $\lim \gamma_{h}=0$; by lemma $2, \Lambda_{h}^{-1}$ exists for $h$ small enough and is uniformly bounded with respect to $h$; furthermore $\sup \left\|\Lambda_{h}^{-1} x-x\right\| \leqq c \gamma_{h}$, i. $\mathrm{e}$. $\delta\left(F_{h}(X), E(X)\right) \leqq c \gamma_{h}$. $x \in F_{h}(x)$
$\|x\|=1$

Proof of theorem 2: Let $S_{h}=\Lambda_{h}^{-1} F_{h}-I: X \rightarrow X ; S_{h}$ is uniformly bounded with respect to $h$ (see proof of theorem 1); from the identity

$$
\left(\hat{A}-\hat{B}_{h}\right) x=\left(A-B_{h}\right) x+S_{h}\left(A-B_{h}\right) x, x \in E(X),
$$

one obtains for $x \in E(X), y \in E^{*}(X)$, since $F_{h} S_{h}=0$,

$$
\begin{gathered}
a\left(\left(\hat{A}-\hat{B}_{h}\right) x, y\right)=a\left(\left(A-B_{h}\right) x, y\right)+a\left(S_{h}\left(A-B_{h}\right) x,\left(I-F_{h}^{*}\right) y\right) ; \\
\left\|\hat{A}-\hat{B}_{h}\right\|_{E(X)} \leqq c \sup _{\substack{x \in E(X), y \in X \\
\|x\|=\|y\|=1}} a\left(\left(\hat{A}-\hat{B}_{h}\right) x, y\right) \\
\leqq c \sup _{\substack{x \in E(X), y \in E^{*}(X) \\
\|x\|=\|y\|=1}} a\left(\left(\hat{A}-\hat{B}_{h}\right) x, y\right) ;
\end{gathered}
$$


for $x \in E(X), y \in E^{*}(X),\|x\|=\|y\|=1$, one has (using lemma 3):

$$
\begin{aligned}
&\left|a\left(S_{h}\left(A-B_{h}\right) x,\left(I-F_{h}^{*}\right) y\right)\right| \leqq c\left\|\left(A-B_{h}\right) x\right\| \cdot\left\|\left(I-F_{h}^{*}\right) y\right\| \leqq c \gamma_{h} \gamma_{h}^{*} \\
& a\left(\left(A-B_{h}\right) x, y\right)= a\left(\left(I-\Pi_{h}\right) A x,\left(I-\Pi_{h}^{*}\right) y\right) \\
&+a\left(\left(I-\Pi_{h}\right) x,\left(I-\Pi_{h}^{*}\right) A^{*} y\right) \\
&+a\left(\left(I-\Pi_{h}\right) x, A^{*}\left(\Pi_{h}^{*}-I\right) y\right) \\
&\left|a\left(\left(A-B_{h}\right) x, y\right)\right| \leqq c \gamma_{h} \gamma_{h}^{*}
\end{aligned}
$$

theorem 2 follows from (2), (3), (4) and (5).

\section{REFERENCES}

1. F. Chatelin, Théorie de l'approximation des opérateurs linéaires, application au calcul des valeurs propres d'opérateurs différentiels et intégraux, Notes de cours, Université de Grenoble, 1977.

2. J. Descloux, N. Nassif and J. Rappaz, Spectral Approximations with Error Bounds for Non-Compact Operators, Rapport du département de mathématiques E.P.F.L., 1977.

3. J. Descloux, N. Nassif and J. Rappaz, On Spectral Approximation, Part 1: The Problem of convergence, this journal.

4. R. D. GrigoriefF, Diskrete Approximation von Eigenwertproblem, Numerische Mathematik; part I: Vol. 24, 1975, pp. 355-374; part II: Vol. 24, 1975, pp. 415-433; part III : Vol. 25, 1975, pp. 79-97.

5. J. E. Osborn, Spectral Approximation for Compact Operators, Math. Comp., Vol. 29, 1975, pp. 712-725.

6. J. RAPPAZ, Approximation of the Spectrum of a Non-Compact Operator Given by the Magnetohydrodynamic Stability of a plasma, Numer. Math., Vol. 28, 1977, pp. 15-24.

7. J. H. Wilkinson, The Algebraic Eigenvalue Problem, Oxford University Press, 1965. 